\title{
Solitary fibrous tumor in the oral cavity: a case report and diagnostic dilemma
}

\author{
Piotr Chomik ${ }^{1}$, Adam Michcik ${ }^{1}$, Igor Michajłowski², Michał Sobjanek², Adam Włodarkiewicz ${ }^{1,2}$ \\ 1Department of Oral and Maxillofacial Surgery, Medical University of Gdansk, Poland \\ Head: Prof. Adam Włodarkiewicz MD, DDS, PhD \\ 2Department of Dermatology, Venereology and Allergology, Medical University of Gdansk, Poland \\ Head: Prof. Roman Nowicki MD, PhD
}

Postep Derm Alergol 2012; XXIX, 5: 395-400

DOI: $10.5114 / p d i a .2012 .31495$

\begin{abstract}
The investigators wish to discuss the diagnostic difficulties, histology and immunohistochemical profile of a solitary fibrous tumor (SFT) based on the presented case, as well as previously reported cases within the oral cavity. A young woman was referred to the Department of Maxillofacial Surgery, Medical University of Gdansk, Poland, due to a considerable palpable mass within the hard palate. Pain and discomfort caused by the tumor's location were the main complaints. A clinical examination revealed a tumor of the right hard palate along the alveolar crest, measuring approximately $5 \mathrm{~cm} \times 3 \mathrm{~cm}$. Panoramic X-ray depicted a bone defect of the alveolar crest around tooth 17. Histological and immunohistochemical evaluation of the biopsy specimen and resected tumor established the diagnosis of SFT. This rare spindle cell neoplasm of mesenchymal origin is typical of serosal sites and approximately 80 cases within the head and neck region have been reported so far. The hard palate is one of the least frequent locations. Approximately $5-20 \%$ of lesions may present features of aggressiveness. The authors wish to emphasize that the diagnosis of SFT is challenging due to a relatively small number of cases reported in the literature, uncertain etiology of the tumor, as well as clinical and histological similarity to other, more frequently occurring benign neoplasms of mesenchymal origin, i.e. hemangiopericytoma, myofibroblastoma, schwannoma, neurofibroma, leiomyoma, as well as inflammatory disorders, especially nodular fasciitis. Radical excision of the tumor is mandatory for effective treatment due to the possibility of recurrence and malignant transformation after subradical resection.
\end{abstract}

Key words: solitary fibrous tumor, oral cavity, hard palate, diagnosis.

\section{Introduction}

A solitary fibrous tumor (SFT) is a spindle cell neoplasm of mesenchymal origin with a particular predilection for serosal sites [1-4]. Klemperer and Rabin described the first case in the visceral pleura in 1931 [5]. Convincingly, due to such a location, it was initially stated that this was a benign variant of mesothelioma [2, 6]. Subsequent ultrastructural research revised this opinion and revealed that SFT originated from interstitial stem cells localized mainly in the soft tissues of various body sites [2, 7]. Despite knowledge of the tumor's origin and reproducible immunohistochemical profile, its biology is still incompletely explained [2, 8].

Most cases express benign behavior, although according to the literature, about $5-20 \%$ of tumors may present features of aggressiveness along with local recurrences, as well as distant metastases to the liver, lungs, bones, mesentery and the retroperitoneal space [7, 9, 10]. Recurrences usually present more advanced histology than primary tumors. On the other hand, some cases of SFT with histological features of malignancy clinically present as benign tumors, which unambiguously indicates that the biology of particular cases cannot be predicted exclusively based on the histological diagnosis [4].

Data in the literature show that SFT within the head and neck comprise only about 3\% of cases, mostly affecting the oral cavity [1]. Tumors within the thyroid gland, larynx, epiglottis, parapharyngeal space, major salivary glands, nasopharynx, paranasal sinuses, orbit, as well as cerebral meninges, are considerably rare [7]. Most patients 
with SFT of the oral cavity present with a slow growing, non-tender mass of various dimensions located within the submucosal tissue $[1,11,12]$.

Regarding the morphology and clinical appearance of SFT, the differential diagnosis should include tumors of the salivary glands, lipoma, mucocoele, vessel malformations, lymphoma, as well as odontogenic abscesses $[9,12,13]$. On the other hand, due to its histological features, SFT should be distinguished from hemangiopericytoma, myofibroblastoma, schwannoma, neurofibroma, leiomyoma and inflammatory diseases, such as nodular fasciitis [9, 10, 14-16].

Herein, the authors present a case of a young woman diagnosed with SFT of the hard palate after initial histological and immunohistochemical analysis of a biopsy specimen. Treatment methods, histological features and an immunohistochemical profile are discussed.

\section{Case report}

A 33-year-old woman was referred to the Department of Maxillofacial Surgery, Medical University of Gdansk, Poland, in order to diagnose and treat a tumor of the hard palate. The lesion had appeared 1.5 years earlier and grew continually. On the day of admission, the patient complained of frequent pain and discomfort due to the tumor's location. The anamnesis revealed hypothyroidism and the patient remained under regular endocrinologic control.

Clinical examination revealed a tumor of the right hard palate along the alveolar crest in close proximity to teeth $15-17$, measuring approximately $5 \mathrm{~cm} \times 3 \mathrm{~cm}$. The lesion presented with a rather soft consistency, was non-tender

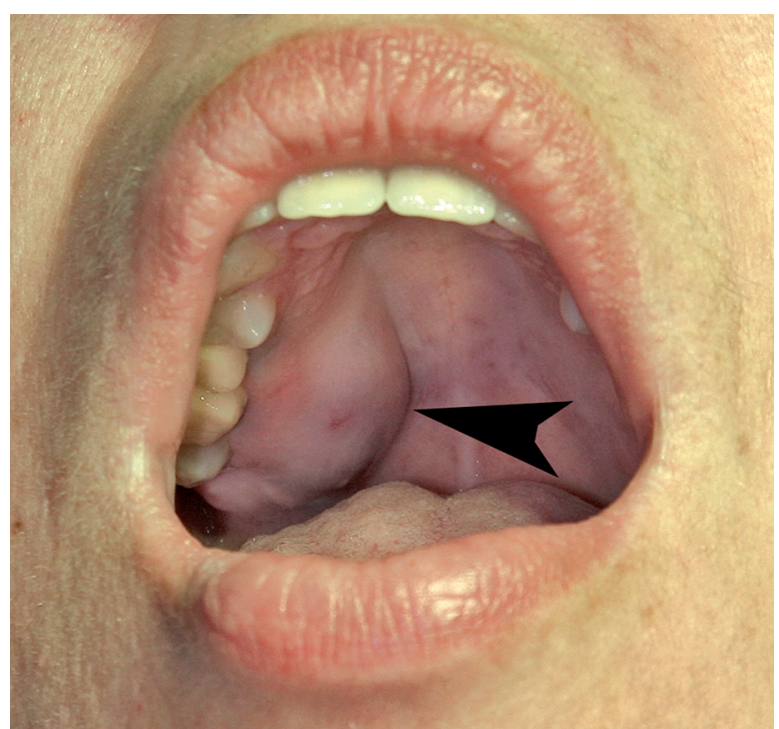

Figure 1. Tumor of the right hard palate along the alveolar crest in close proximity to teeth 15-17 (arrowhead). The covering mucosa tends to be slightly paler compared to the surrounding tissues during palpation, and was covered with mucosa, which tended to be slightly paler compared to the surrounding tissue (Figure 1). Panoramic $X$-ray revealed a bone defect of the alveolar crest around tooth 17 (Figure 2).

Microscopic evaluation of the biopsy specimen stated: spindle cell neoplasm with slight nuclear pleomorphism and the presence of vessels, part of which express a sharpened ("staghorn") shape. Phenotype: CD34+, S100+, SMA-, GFAP-, CK AE 1/3-, NF-, EMA-. Overall, the microscopic picture is most typical of a solitary fibrous tumor.

Following such a diagnosis, the tumor was resected along with a $1 \mathrm{~cm}$ margin of clinically unsuspected tissue under general anesthesia (Figures 3, 4). Pathologic evaluation of the excised lesion confirmed the initial diagnosis: solitary fibrous tumor; phenotype: CD34+, SMA-, S100-, CD99+, bcl2+, CK AE 1/3-. The patient remains under regular clinical follow-up, currently without signs of recurrence.

\section{Discussion}

Solitary fibrous tumor typically occurs between the fourth and eighth decade of life with equal frequency in both sexes $[11,12,15]$. The etiopathogenesis of the tumor remains unclear. Two theories have been advocated in the literature to clarify this issue [7]. The first one assumes multidirectional differentiation of fibroblasts and mesenchymal multipotential cells in connective tissue [7]. The second one relates to the existence of specialized cells, capable of differentiation towards mesothelium [7]. Nevertheless, most evidence advocates the theory of the mesenchymal origin of SFT [7, 17].

Moreover, lesions appearing in the oral cavity have not been accompanied by systemic symptoms in any of the reported cases. On the other hand, these symptoms are typical of SFT at serosal sites, such as the pleura $[4,15]$.

Among approximately 80 cases of SFT in the oral cavity, the most frequent sites were the tongue and buccal tissues, which may indicate irritation as a potential etiologic factor for this tumor arising in these particular locations $[1,15]$.

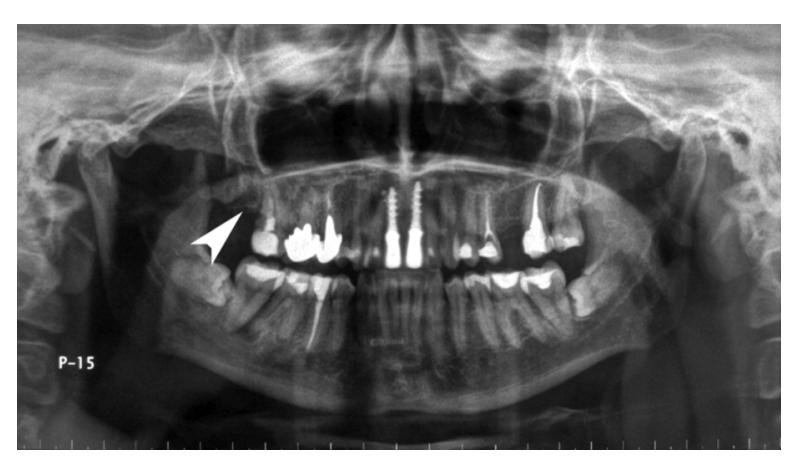

Figure 2. Panoramic X-ray of the patient revealed a bone defect of the alveolar crest around tooth 17 (arrowhead) 


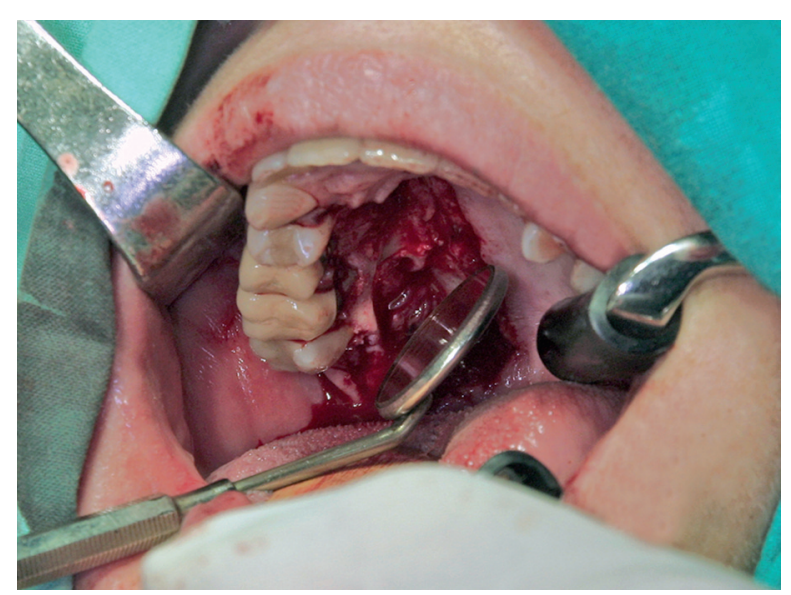

Figure 3. Intraoperative view directly after tumor resection. The bone defect is visible within the tumor site

Concerning the similarity to other more frequently occurring neoplasms of mesenchymal origin, both histological and immunohistochemical diagnosis may be challenging even to the experienced pathologist. Because of this, Chan et al. [18] established diagnostic criteria which enable establishing a diagnosis of SFT. They include clinical, histological and immunohistochemical characteristics of the tumor, among others clinically fair demarcation of the lesion from surrounding tissues, alternating hypercellular foci and hypocellular sclerotic foci, blandlooking, short and spindly or ovoid cells with scanty and poorly defined cytoplasm, few mitotic figures (<4/10 HPF), haphazard, storiform or fascicular arrangement of spindly cells (a so-called "patternless pattern"), intimate intertwining of thin or thick collagen fibrils with spindly cells, and immunohistochemical positivity for CD34, which is obligatory to confirm the diagnosis of extrapleural SFT $[15,16,19]$.

Additionally, the lack of encapsulation of the tumor, as well as small cell dimensions and cell membrane disturbances may be observed. The cytoplasm presents a weak eosinophilic reaction and spindly nuclei contain diffuse chromatin and small nucleoli $[7,15,16]$. Fairly numerous blood vessels presenting a typical "staghorn pattern" are common, which is particularly seen among other features mentioned above in the microscopic specimen of the presented case (Figure 5), as well as more or less abundant infiltrates of mast cells.

As mentioned above, histology of SFT is typical of most neoplasms of mesenchymal origin, thus immunohistochemical labeling is obligatory for a correct diagnosis. The vast majority of SFTs express a positive reaction for CD34 (Figure 6) and vimentin [7, 9]. The same reaction may be observed for CD99 and bcl-2, although this is not the rule [9]. At times, positive staining for factor XIIla may be present [20]. A negative reaction is common for S-100 protein (Figure 7 ), cytokeratin (Figure 8), desmin and

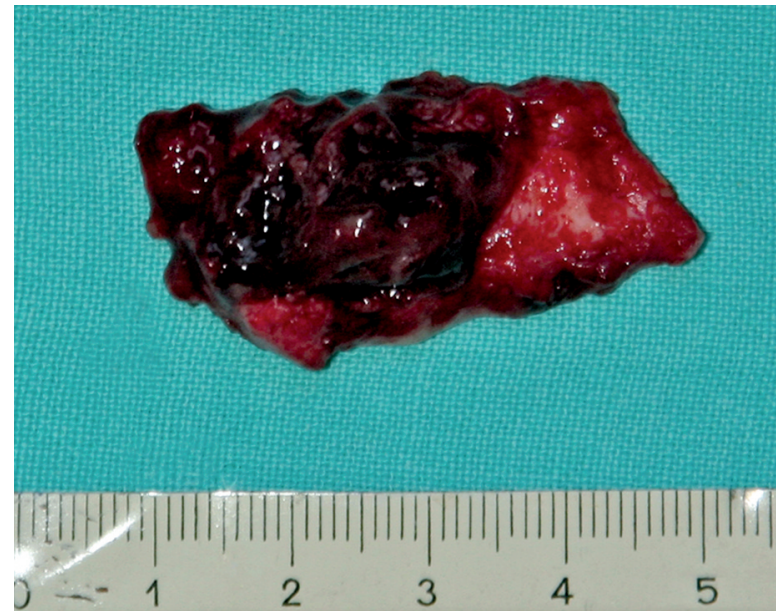

Figure 4. The tumor mass directly after resection

actin [12]. In the presented case, immunohistochemical evaluation of the biopsy specimen demonstrated positive staining for S-100 protein; nevertheless, the resected tumor presented an unequivocally negative reaction. Eventually, it was stated that the biopsy specimen examination revealed a false positive result for S-100 protein. This molecule is common in nerve sheaths, thus positive staining is typical of lesions of neuronal origin, for example schwannoma or neurofibroma.

Five to twenty percent of SFT cases have been shown to display both microscopic and clinical characteristics of malignancy $[1,4,7]$. Histologically, these tumors tend to contain greater amounts of cells with focally observed significant alterations, features of necrosis, numerous mitotic figures, as well as infiltrated borders [7]. Differentiation between benign and malignant variants on the microscopic level is primarily based on the presence

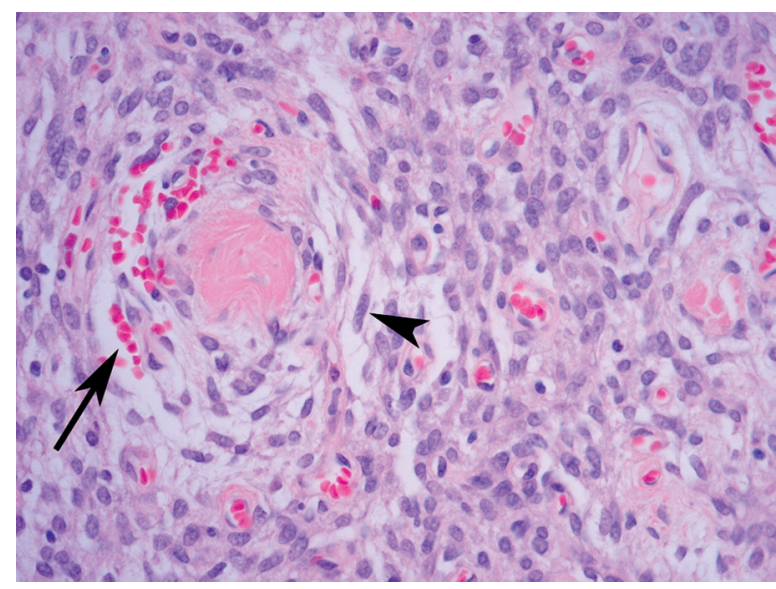

Figure 5. With hematoxylin and eosin staining, haphazardly arranged spindly cells are visible (arrowhead). Bundles of collagen fibers and fairly numerous blood vessels presenting the typical "staghorn pattern" (arrow) are also seen. Magnification 100x 


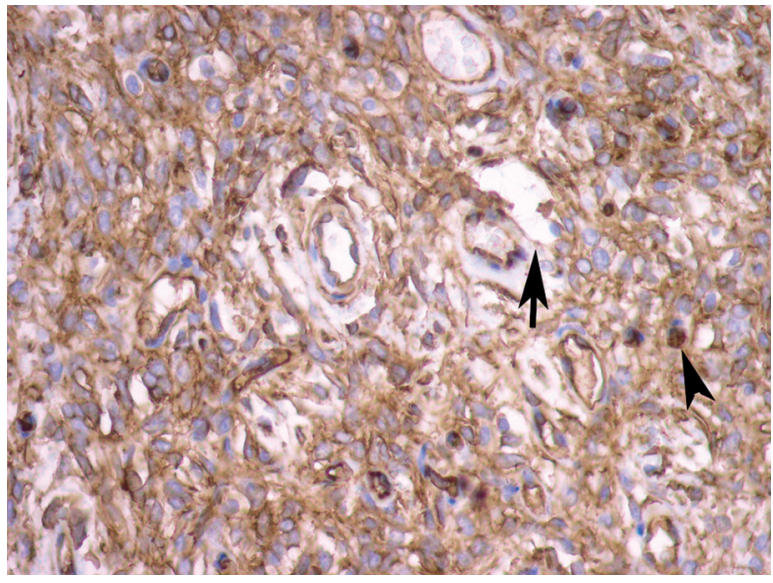

Figure 6. Immunopositive CD34 reaction expressed by tumor cells (arrowhead) and negative reaction within vessel walls (arrow). Magnification 100x

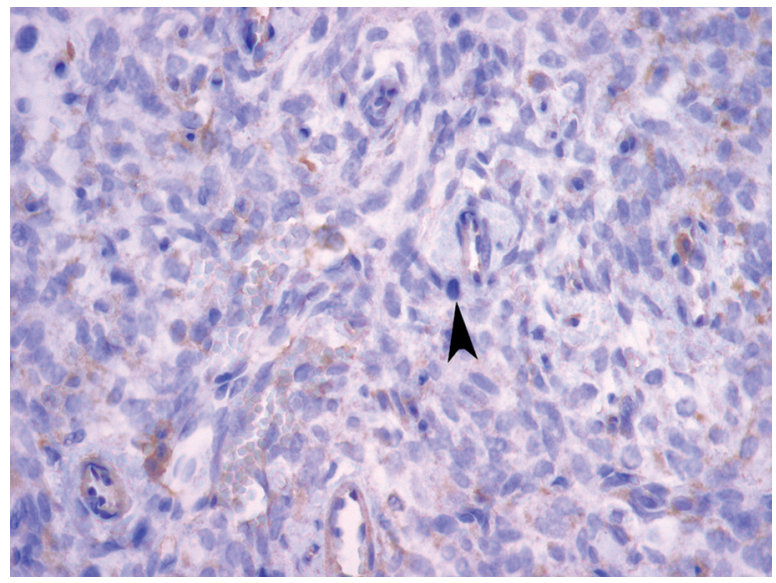

Figure 7. SFT cells are typically immunonegative for S-100 protein (arrowhead), which is specific of nerve sheaths. Magnification 100x

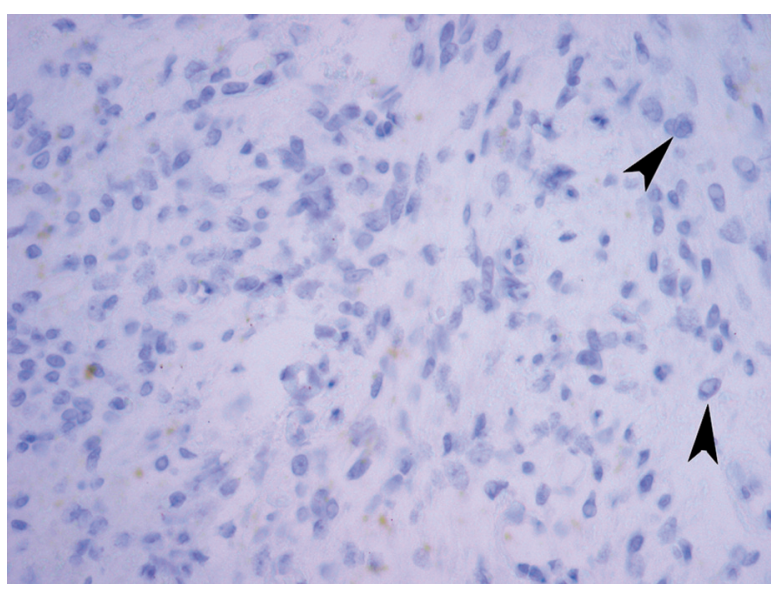

Figure 8. SFT cells demonstrate negative immunohistochemical reactions for CKAE 1 and CKAE 3 (arrowheads). Magnification $100 x$ and number of mitotic figures which are sparse or even absent in benign cases, as well as nuclear alterations typical of malignant cases [7]. Fusconi et al. [6] have also described the presence of sarcoma-like foci within SFT as well as the development of sarcoma at the location of previously resected SFT as a malignancy criterion of the latter. Features of clinical malignancy include first of all local recurrences, which may occur even a few years after total resection of the primary tumor, distant metastases, particularly to the liver, lungs, bones and mesentery, as well as the retroperitoneal space [9]. Especially important is the fact that recurrences usually present a more advanced phenotype compared to primary lesions.

Total surgical resection along with macroscopically unsuspected tissue margins is the treatment of choice in SFT cases $[1,4,7,9]$. Reports of postoperative radiotherapy and chemotherapy with adriamycin and dacarbazine in cases of large tumors with positive margins are available in the literature [7, 9]. In the reported case, total surgical resection, which was confirmed by histological examination of the excised tissue specimen, was considered to be sufficient treatment.

Differential diagnosis of SFT relates to other mesenchymal tumors, including hemangiopericytoma, myofibroblastoma, schwannoma, neurofibroma, leiomyoma, as well as inflammatory disorders such as nodular fasciitis $[9,10,12,14-16]$. The most challenging issue is the differentiation between SFT and hemangiopericytoma due to very similar histological and immunohistochemical profiles. It is believed that hemangiopericytoma is more homogenous. It also contains fewer collagen fibers and more cells compared to SFT [12]. Positive CD34 staining tends to be more focal and less regular in cases of hemangiopericytoma [12].

Myofibroblastoma histologically presents uniform spindly cells arranged in short bundles. Moreover, molecular evaluations have revealed that these cells differentiate into both fibroblasts and smooth muscle cells, which results in positive immunohistochemical staining towards smooth muscle actin (SMA). As mentioned above, such a reaction is negative in cases of SFT [10].

Spindle cell neoplasms of neuronal origin, including schwannoma and neurofibroma, display some clinical and immunohistochemical attributes similar to SFT. These lesions are typically fairly well circumscribed from surrounding tissues, which they owe to the presence of a capsule around the tumor. On the other hand, despite its fair demarcation from surrounding tissues, SFT is an unencapsulated tumor [10]. Distinct positive staining for S-100 protein, which is typical of nerve sheaths, is a general immunohistochemical hallmark of neuronal tumors. As mentioned above, SFT displays a negative reaction for S-100 protein, which disqualifies its neuronal origin [14].

Leiomyoma is characterized by more fascicular and regular arrangements of spindly cells compared to SFT [10]. Ultrastructural findings present the differentia- 
tion of these cells towards smooth muscle, thus immunohistochemical staining for SMA is positive. Additionally, immunoreactions towards CD34 and bcl-2 are negative in cases of leiomyoma [10]. In conclusion, differentiation between SFT and leiomyoma on an immunohistochemical level should not be a challenge.

Nodular fasciitis (NF) is a separate inflammatory entity which should be taken into consideration in the differential diagnosis of SFT. Clinically, both disorders represent fair circumscription from surrounding tissues. Moreover, spindly cells are common for both lesions microscopically. On the other hand, in cases of SFT, the presence of mature bundles of collagen fibers can be found, which are absent in NF. Moreover, a large number of relatively loosely and regularly arranged cells is typical of NF [21]. Immunohistochemical evaluation reveals the most evident differences between these two entities. In cases of NF, cells stain positive for SMA, whereas positive immunostaining for CD34 is visible only in close proximity to vessel walls. On the other hand, SFT stains unambiguously negative for SMA, whereas staining for CD34 is intensively positive within the entire lesion [21].

In order to examine the pathology of SFT entirely, Manor and Bodner [22] analyzed chromosomal aberrations within their case and summarized similar analyses of other researchers. So far, it has been proven that chromosomal aberrations typical of SFT may be characterized by additional chromosomes 2, 7, 8, 9, 10, 18 and 21 in tumor cells, additional arms of chromosomes 12p, $12 q$ and 15q, loss of arms 4q, 5p, 6, 9p, 13q, 15q, and 20p, loss of entire chromosomes 6, 10, 17, 21, 22 and X [23], as well as complex translocations within chromosomes 1, 17 and 18 [22]. It has been also stated that the most frequent chromosomal aberration in SFT is the loss of arm 13q [22]. The significance of this abnormality and its influence on the development and biology of the tumor has not been established yet.

Ultrastructural analysis of SFT performed by Ide et al. [20] suggested that it may be responsible for intensive angiogenesis at a particular stage of its development, which results in the typical "staghorn pattern" of vessel arrangement in the microscopic evaluation of SFT.

In summary, we state that in spite of the fairly wellknown histological and immunohistochemical profile of SFT, its etiopathogenesis is still insufficiently explained. Consequently, the clinical behavior of the tumor and its biology are extraordinarily difficult to predict, and local recurrences as well as distant metastases are possible even a few years after histologically proven radical resection. With an awareness of these facts, patients must remain under long-term follow-up after the treatment has been completed. Further molecular studies in order to accurately explore the biology of SFT and application of the most effective treatment at an early stage are crucial priorities.

\section{References}

1. Amico P, Colella G, Rossiello R, et al. Solitary fibrous tumor of the oral cavity with a predominant leiomyomatous - like pattern: a potential diagnostic pitfall. Pathol Res Pract 2010, 206: 499-503.

2. Swelam WM, Cheng J, Ida-Yonemochi H, et al. Oral solitary fibrous tumor: a cytogenetic analysis of tumor cells in culture with literature review. Cancer Genet Cytogen 2009; 194: 75-81.

3. Harada T, Matsuda H, Maruyama R, Yoshimura Y. Solitary fibrous tumours of the lower gingiva: a case report. Int J Oral Maxillofac Surg 2002; 31: 448-50.

4. El-Sayed IH, Eisele DW, Yang TL, lezza G. Solitary fibrous tumor of the retropharynx causing obstructive sleep apnea. Am J Otolaryng 2006; 27: 259-62.

5. Klemperer P, Rabin CB. Primary neoformations of the pleura: a report of five cases. Arch Pathol 1931; 11: 385.

6. Fusconi M, Ciofalo A, Greco A, et al. Solitary fibrous tumor of the oral cavity: case report and pathologic consideration. J Oral Maxillofac Surg 2008; 66: 530-4.

7. Gonzalez-Garcia R, Gil-Diez Usandizaga J, Hyun Nam S, et al. Solitary fibrous tumour of the oral cavity with histological features of aggressiveness. Br J Oral Max Surg 2006; 44 543-5.

8. Karakis GP, Sin B, Tutkak H, et al. Genetic aspect of venom allergy: association with HLA class I and class II antigens. Ann Agric Environ Med 2010; 17: 119-23.

9. Shnayder Y, Greenfield BJ, Oweity T, DeLacure MD. Malignant solitary fibrous tumor of the tongue. Am J Otolaryngol 2003; 24: 246-9.

10. Wu SL, Vang R, Clubb Jr FJ, Connelly JH. Solitary fibrous tumor of the tongue: report of a case with immunohistochemical and ultrastructural studies. Ann Diagn Pathol 2002; 6: 168-71.

11. Correia Jham B, Porcaro Salles JM, Arantes Soares JM, et al. Solitary fibrous tumour of the buccal mucosa: case report and review of the literature. Br J Oral Max Surg 2007; 45: 323-5.

12. Shine N, nor Nurul Khasri M, Fitzgibbon J, O`Leary G. Solitary fibrous tumor of the floor of the mouth: case report and review of the literature. Ear Nose Throat J 2006; 85: 437-9.

13. Talacko AA, Aldred MJ, Sheldon WR, Hing NR. Solitary fibrous tumour of the oral cavity: report of two cases. Pathology 2001; 33: 315-8.

14. Hirano M, Tanuma J, Shimoda T, et al. Solitary fibrous tumor in the mental region. Pathol Int 2001; 51: 905-8.

15. Alawi F, Stratton D, Freedman PD. Solitary fibrous tumor of the oral soft tissues. A clinicopathologic and immunohistochemical study of 16 cases. Am J Surg Pathol 2001; 7 : 900-10.

16. Perez-Ordonez B, Koutlas IG, Strich E, et al. Solitary fibrous tumor of the oral cavity. An uncommon location for a ubiquitous neoplasm. Oral Surg Oral Med Oral Pathol Oral Radiol Endod 1999; 87: 589-93.

17. Guerra MF, Amat CG, Campo FR, Perez JS. Solitary fibrous tumor of the parotid gland. A case report. Oral Surg Oral Med Oral Pathol Oral Radiol Endod 2002; 94: 78-82.

18. Gray PB, Miller AS, Loftus MJ. Benign fibrous histiocytoma of the oral/perioral regions: report of a case and review of 17 additional cases. J Oral Maxillofac Surg 1992; 50: 1239-42.

19. Kurihara K, Mizuseki K, Sonobe J, Yanagihara J. Solitary fibrous tumor of the oral cavity. A case report. Oral Surg Oral Med Oral Pathol Oral Radiol Endod 1999; 87: 223-6. 
20. Ide F, Obara K, Mishima K, et al. Ultrastructural spectrum of solitary fibrous tumor: a unique perivascular tumor with alternative lines of differentiation. Virchows Arch 2005; 446 : 646-52.

21. Eversole LR, Christensen R, Ficarra G, et al. Nodular fascitis and solitary fibrous tumor of the oral region. Tumors of fibroblast heterogeneity. Oral Surg Oral Med Oral Pathol Oral Radiol Endod 1999; 87: 471-6.

22. Manor E, Bodner L. Chromosomal aberrations in oral solitary fibrous tumor. Cancer Genet Cytogen 2007; 174: 170-2.

23. Martin AJ, Summersgill BM, Fisher C, et al. Chromosomal imbalances in meningeal solitary fibrous tumor. Cancer Genet Cytogen 2002; 135: 160-4. 